\title{
On Dynamic Survival Past Extropy Properties
}

\author{
Zohreh Pakdaman* and Majid Hashempour \\ University of Hormozgan
}

Received: 8/22/2020 Approved: 10/29/2020

\begin{abstract}
This paper deals with the dynamic survival past extropy as a measure of uncertainty in the past lifetime distributions. We introduce a quantile version of the extropy function in past lifetime. Various properties of the proposed measure are obtained. Additionally, some stochastic comparisons and bounds are derived and the performance of the dynamic survival past extropy of parallel and series system is studied as well.
\end{abstract}

Keywords. Cumulative extropy; order statistics; past lifetime; quantile function; stochastic orders.

MSC 2010: 62N05; 62F10.

\section{Introduction}

Entropy as a widely used concept in many scientific disciplines such as physics, probability, statistics, communication theory, information theory, economics has been firstly introduced by Shannon (1948). In information theory, entropy is a measure of the uncertainty associated with a random variable. Shannon's entropy represents the absolute limit on the best possible lossless compression of any communication. The interested reader may refer to Cover and Thomas (2012) for further details. Let $X$ is non-negative

${ }^{*}$ Corresponding author 
random variable with an absolutely continuous cumulative distribution function (cdf) $F$, probability density function (pdf) $f$ and the survival function (sf) $\bar{F}(x)=1-F(x)$. Shannon entropy of $X$ is defined as

$$
H(X)=-E(\log f(X))=-\int_{0}^{+\infty} f(x) \log f(x) d x,
$$

in which, "log" means the natural logarithm. Recently, Lad et al. (2015) proposed an alternative measure of uncertainty of a random variable called extropy. The term extropy, as an antonym to entropy, had been used earlier in academic literature as well. Extropy is the extent of a living or organizational system's intelligence, functional order, vitality, energy, life, experience, and capacity and drives for improvement and growth. It is not a rigorously defined technical term in the philosophy of science. In a metaphorical sense, extropy can simply express the opposite of entropy. Lad et al. (2015) defined the extropy of non-negative random variable $X$ as

$$
\begin{aligned}
J(X) & =-\frac{1}{2} \int_{0}^{+\infty} f^{2}(x) d x \\
& =-\frac{1}{2} \int_{0}^{1} f\left(F^{-1}(u)\right) d u .
\end{aligned}
$$

The properties of this measure such as the maximum extropy distribution and statistical applications were presented in their work. Yang et al. (2018) studied the relations between extropy and variational distance. They determined the distribution which attains the minimum or maximum extropy among these distributions within a given variation distance from any given probability distribution. Also, Qiu et al. (2019) explored an expression of the extropy of a mixed system's lifetime. Jahanshahi et al. (2019) introduced an alternative measure of uncertainty of the random variable $X$ which they called it cumulative residual extropy $(\mathrm{CRJ})$ as

$$
C R J(X)=-\frac{1}{2} \int_{0}^{+\infty} \bar{F}^{2}(x) d x .
$$

Moreover, they studied some properties of the aforementioned information measure. Qiu and Jia (2018) proposed residual extropy to measure residual 
uncertainty of the random variable $X$ as follows

$$
\mathrm{J}_{t}(X)=-\frac{1}{2} \int_{t}^{+\infty} f_{t}^{2}(x) d x
$$

where $f_{t}(x)=f(x) / \bar{F}(t), x>t$. The $\mathrm{J}_{t}(X)$ is suitable to measure information when uncertainty is related to the future. For more recent works on extropy, one can also refer to Alizadeh and Jarrahiferiz (2019), Noughabi and Jarrahiferiz (2020), Qiu and Jia (2018), Jose and Sathar (2019), Qiu (2017), and Krishnan et al. (2020) and the references therein.

In this paper, we introduce a new measure of uncertainty that will be called dynamic survival past extropy (DSPJ). We consider the concept DSPJ which relates to the uncertainty on the past lifetime of a system. The dynamic survival past extropy for the past lifetime of the random variable $X$ is defined as

$$
\operatorname{DSPJ}_{F}(t)=-\frac{1}{2} \int_{0}^{t} \bar{F}_{t}^{2}(x) d x
$$

where $\bar{F}_{t}(x)=\frac{F(x)}{F(t)}, x<t$. For the special case $t=\infty$, we define the cumulative extropy (CJ) of random variable $X$ as

$$
\mathrm{CJ}_{\mathrm{F}}=-\frac{1}{2} \int_{0}^{+\infty} F^{2}(x) d x .
$$

The goal of this paper is to enquire about some new form of survival and past extropy functions using the underlying distribution function and quantile function procedures. The quantile version of various entropies was presented with the purposes of providing alternative ways, new results and different methods of stochastic comparisons. For details we refer to Sunoj and Sankaran (2012), Yu and Wang (2013), Nanda et al. (2014), Sunoj et al. (2017) and Qiu (2019). The quantile function strategy allows us to discover some results which are hard to obtain using the definition of survival and past extropies in the form of the underlying distribution function.

The structure of the paper is as follows. In Section 2, some relevant definitions are presented. Moreover, the features of dynamic survival past extropy and its relation with reversed hazard rate function are evaluated in Section 3. The quantile-based approach for the dynamic survival past extropy is studied in Section 4 in details and further some stochastic comparisons are investi- 
gated in this section. Additionally, the cumulative extropy as the special case of the dynamic survival past extropy is studied. Finally, the behaviour of the dynamic survival past extropy of some order statistics is evaluated and some results are established.

\section{Some Definitions}

This section devoted to the review of some definitions about the stochastic orders and quantile function. More details of stochastic orders can be found in Shaked and Shanthikumar (2007).

Definition 1. Let the cdf of random variable $X$ is $F(x)$, then the quantile function corresponding to cdf $F(x)$ is defined by

$$
Q(u)=\inf \{x \mid F(x) \geq u\}, \quad 0 \leq u \leq 1,
$$

and $q(u)=\frac{d Q(u)}{d u}$ is the quantile density function. Also, the reversed hazard quantile function of the random variable $X$ is $\lambda_{Q}(u)=\frac{1}{u q(u)}$.

Definition 2. Let $X$ and $Y$ be two random variables with cdfs $F$ and $G$ and pdfs $f$ and $g$, respectively. Assume the ratios below are well defined. Then $X$ is said to be smaller than $Y$ in the sense of:

(i) usual stochastic order (denoted by $X \leq_{s t} Y$ or $F \leq_{s t} G$ ) if $\bar{F}(x) \leq \bar{G}(x)$ for all $x$;

(ii) hazard rate order (denoted by $X \leq_{h r} Y$ or $F \leq_{h r} G$ ) if $\frac{\bar{G}(x)}{\bar{F}(x)}$ is increasing in $x$;

(iii) reversed hazard quantile order (denoted by $X \leq_{R H Q} Y$ or $F \leq_{R H Q} G$ ) if $\lambda_{Q_{X}}(u) \leq \lambda_{Q_{Y}}(u)$ for all $u \in(0,1)$;

(iv) dynamic survival past quantile extropy (denoted by $X \leq_{D S P Q J} Y$ ) if $D S P J_{Q_{X}}(u) \leq D S P J_{Q_{Y}}(u)$ for all $0 \leq u \leq 1$;

(v) dynamic survival past extropy (denoted by $X \leq{ }_{D S P J} Y$ ) if $D S P J_{F}(t) \leq$ $D S P J_{G}(t)$ for all $0 \leq t \leq 1$.

\section{Dynamic Survival Past Extropy}

In a real life situation, one may be interested in getting uncertainty about the history of the system. It is logical to assume that in some realistic circumstances, the uncertainty is not unavoidably related to the future, but 
somehow it can be pointed to the past as well. For instance, a system which is observed only at certain pre-assigned inspection times is found to be down, then the uncertainty of the system life relies on the past, i .e., on which instant it has actually failed. For a non-negative random variable $X$ with an absolutely continuous cdf $F$, we define the dynamic survival past extropy (DSPJ) as

$$
\begin{aligned}
\operatorname{DSPJ}_{F}(t) & =-\frac{1}{2} \int_{0}^{t}\left(\frac{F(x)}{F(t)}\right)^{2} d x \\
& =-\frac{1}{2 F^{2}(t)} \int_{0}^{F(t)} \frac{u^{2}}{f\left(F^{-1}(u)\right)} d u .
\end{aligned}
$$

In the following, the relationship between the dynamic survival past extropy and the reversed hazard rate is presented.

Theorem 1. Let $\operatorname{DSPJ}_{F}(t)$ and $\lambda(t)$ be the dynamic survival past extropy and reversed hazard rate of non-negative random variable $X$ with an absolutely continuous cdf $F$, respectively. Then, we have

$$
\lambda(t)=\frac{2 \operatorname{DSPJ}_{F}^{\prime}(t)+1}{-4 \operatorname{DSPJ}_{F}(t)}
$$

Proof. It is quite apparent from Equation (6) that

$$
\begin{aligned}
\frac{d \operatorname{DSPJ}_{F}(t)}{d t} & =\frac{-1}{2}\left[1-2 \int_{0}^{t} F^{2}(x) F^{-3}(t) f(t) d t\right] \\
& =\frac{-1}{2}-2 \operatorname{DSPJ}_{F}(t) \lambda(t)
\end{aligned}
$$

where $\lambda(t)=\frac{f(t)}{F(t)}$. Therefore, the proof is complete.

Theorem 2. The relationship $\operatorname{DSPJ}_{F}(t)=-\frac{K}{\lambda(t)}$ where $K$ is a non-negative constant holds for all $t>0$ if and only if $F$ be

(i) uniform distribution $F(x)=\frac{x-a}{b-a}, a \leq x \leq b$ if $K=\frac{1}{6}$.

(ii) power I distribution $F(x)=\left(\frac{x}{\alpha}\right)^{\beta}, 0 \leq x \leq \alpha, \beta \geq 0$ if $K=\frac{\beta}{2(2 \beta+1)}$, $0<K<1$.

(iii) power II distribution $F(x)=\left(\frac{x+1}{b+1}\right)^{c},-1 \leq x \leq b, c \geq 0$ if $K=\frac{c}{2(2 c+1)}$, $0<K<1$.

(iv) type III extreme value $F(x)=e^{c(x-b)},-\infty<x \leq b, c>0$ if $K=\frac{1}{4}$. 
Table 1. $\operatorname{DSPJ}_{F}(t)$ for some distributions.

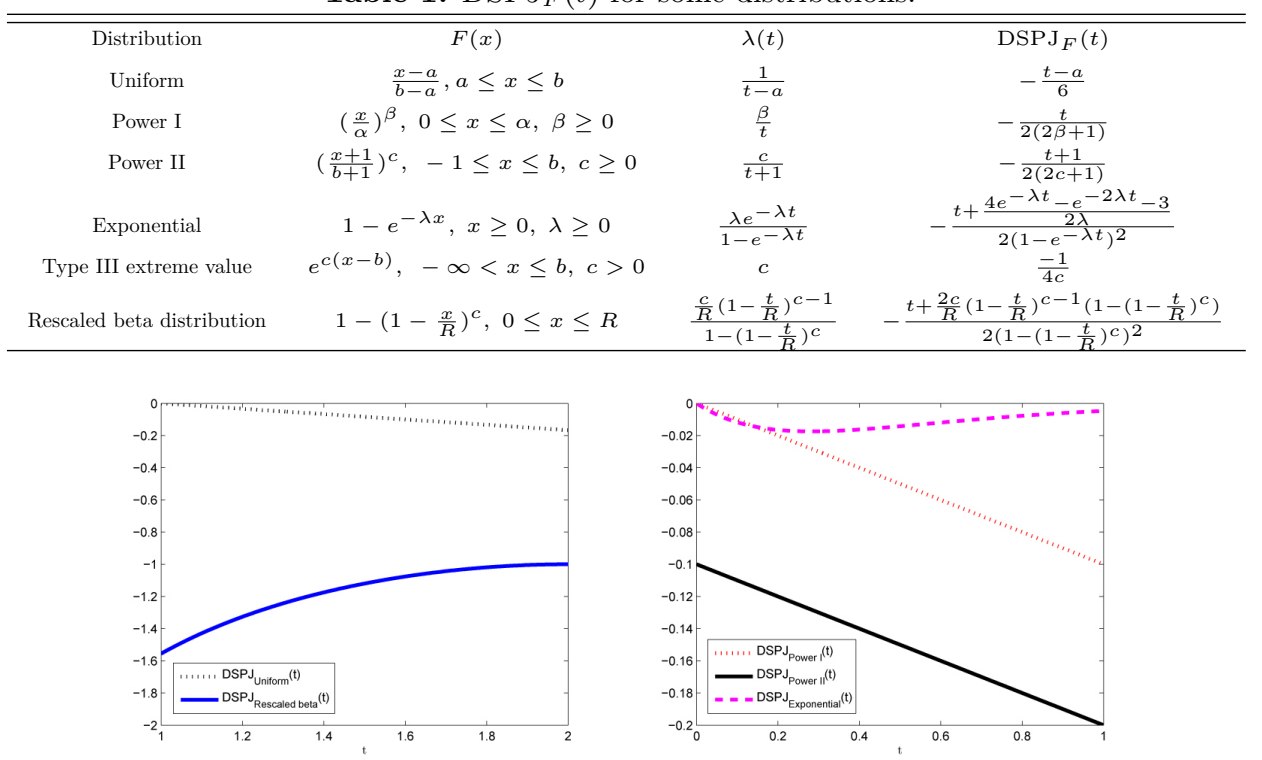

Figure 1. Plot of cumulative past extropy for the distributions in Table (1).

Proof. The result follows immediately from Table 1.

For the distributions in Table 1, the dynamic survival past extropy functions have been depicted in Figure 1. Assume that the location, scale and shape parameters in the aforementioned distributions have values 1, 2 and 3 , respectively.

Theorem 3. Let $X$ be the random variable with support $[-1, b]$. Then the relation $\operatorname{DSPJ}_{F}(t)=-\frac{K}{\lambda(t)}$ where $K>\frac{1}{4}$ is a constant, holds for all $t \in(-1, b]$, if and only if $X$ follows Power II distribution as

$$
F(x)=\left(\frac{x+1}{b+1}\right)^{c}, \quad-1 \leq x \leq b, c \geq 0 .
$$

Proof. The necessary part follows from Table 1 , where $K=\frac{c}{2(2 c+1)}$. From relation (7), we have $\frac{\lambda^{\prime}(t)}{\lambda^{2}(t)}=\frac{4 K-1}{2 K}$. By doing some calculation we obtain 
$\lambda(t)=\frac{1}{\frac{4 K-1}{2 K} t+d}$ with $d>0$ and therefore we get

$$
F(x)=e^{-\int_{x}^{b} \lambda(t) d t}=\left(\frac{x+\frac{2 d K}{4 K-1}}{b+\frac{2 d K}{4 K-1}}\right)^{\frac{2 K}{4 K-1}} .
$$

By placing $K=\frac{1}{4-2 d}$ and $d=\frac{4 K-1}{2 K}$, Equation (10) results in Equation (9).

It should be mentioned that, for $t=\infty$, we have $\operatorname{DSPJ}_{F}(\infty)=C J_{F}$. Let $X$ is a non-negative random variable with an absolutely continuous cdf $F$. We define the CJ as

$$
\begin{aligned}
\mathrm{CJ}_{F} & =-\frac{1}{2} \int_{0}^{+\infty} F^{2}(x) d x \\
& =-\frac{1}{2} \int_{0}^{1} \frac{u^{2}}{f\left(F^{-1}(u)\right)} d u .
\end{aligned}
$$

Corollary 1. For random variable $X$ with $\mathrm{CJ}_{F}$, we have

$$
\mathrm{CJ}_{F}=-\frac{1}{2}\left[\int_{0}^{\infty} F(x) d x-E_{t}\left(H_{F}(t)\right)\right],
$$

where $E_{t}\left(H_{F}(t)\right)=\int_{0}^{+\infty} f(t) \int_{0}^{t} F(x) d x d t$ and $H_{F}(t)=\int_{0}^{t} F(x) d x$.

Proof. From Equation (11), we obtain

$$
\begin{aligned}
\mathrm{CJ}_{F} & =-\frac{1}{2} \int_{0}^{+\infty} F^{2}(x) d x \\
& =-\frac{1}{2} \int_{0}^{+\infty} F(x)\left(\int_{0}^{x} f(t) d t\right) d x \\
& =-\frac{1}{2} \int_{0}^{+\infty} f(t)\left(\int_{0}^{\infty} F(x) d x-\int_{0}^{t} F(x) d x\right) d t .
\end{aligned}
$$

Therefore, from Equation (13) the proof is completed.

The mean past lifetime (MPL) of random variable $X$ at time $t$, which we denote by $M_{F}(t)$, is defined as $M_{F}(t)=\frac{\int_{0}^{t} F(x) d x}{F(t)}$. Using the relation $M_{F}(t)=\frac{H_{F}(t)}{F(t)}$, we can obtain another alternative expression of the CJ, that 
is expressed in terms of MPL as

$$
\mathrm{CJ}_{F}=-\frac{1}{2}\left[\int_{0}^{\infty} F(x) d x-E_{t}\left(F(t) M_{F}(t)\right)\right] .
$$

It should be noted that if the expected value of $X$ exists, then the cumulative Extropy is $-\infty$ and this demonstrates the necessity to study the quantilebased dynamic survival past extropy approach.

\section{Dynamic Survival Past Quantile Extropy}

In the field of quantile functions, we can present the the dynamic survival past quantile extropy as follows

$$
\operatorname{DSP}_{Q}(u)=-\frac{1}{2 u^{2}} \int_{0}^{u} p^{2} q(p) d p
$$

By differentiating from Equation (15), we obtain

$$
q(u)=-2 D S P J_{Q}^{\prime}(u)-\frac{4}{u} D S P J_{Q}(u) .
$$

Equation (16) shows that the baseline distribution is characterized by $D S P J_{Q}(u)$ and it can be used to make the new quantile functions based on the functional forms of $D S P J_{Q}(u)$. Also, $D S P J_{Q}(u)$ specifies the reversed hazard rate as follows

$$
\lambda_{Q}(u)=\frac{1}{-2 u D S P J_{Q}^{\prime}(u)-4 D S P J_{Q}(u)} .
$$

Example 8. Consider the linear mean residual quantile function family of distribution (Midhu et al. (2013)) is identified by

$$
Q(u)=-(c+\mu) \log (1-u)-2 c u, \mu>0,-\mu \leq c<\mu, 0 \leq u \leq 1 .
$$

which includes the exponential and uniform distributions and approximates several continuous distributions closely. In the present case, the cumulative past quantile extropy is

$$
\operatorname{DSPJ}_{Q}(u)=\frac{c+\mu}{2}\left(\frac{1}{2}+\frac{1}{u}+\frac{1}{u^{2}} \log (1-u)\right)+\frac{c u}{2} .
$$


It should be mentioned that the reversed hazard rate of the aforementioned quantile function is

$$
\lambda_{Q}(u)=\left(\frac{(c+\mu) u}{1-u}-2 c u\right)^{-1} .
$$

Corollary 2. Let $X$ be an absolutely continuous non-negative random variable. Then $D S P J_{Q}(u)$ is the linear of the form $D S P J_{Q}(u)=A+B u, A<0$ and $B<0$ if and only if $\lambda_{Q}(u)$ is the inverse linear function.

Proof. From Equation (17), we have

$$
\lambda_{Q}(u)=\frac{1}{-6 B u-4 A}=\frac{1}{a+b u}, a=-4 A, b=-6 B,
$$

which is a hazard quantile function when $A<0$ and $B<0$. On the contrary, Assume that $\lambda_{Q}(u)=\frac{1}{a+b u}, a>0$ and $b>0$, therefore from Equation (17) we have

$$
a+b u=-2 u D S P J_{Q}^{\prime}(u)-4 D S P J_{Q}(u) .
$$

By integrating factor $u^{2}$ from Equation (17), we conclude that

$$
D S P J_{Q}(u)=-\frac{a}{4}-\frac{b}{6} u+\frac{c}{u^{2}} .
$$

It satisfies the Equation (22) when $c=0$.

Distributions with inverse linear reversed hazard quantile function include the uniform, power I and power II distributions. Thus, Result 2 provides a characterization of the inverse linear reversed hazard quantile function family in terms of linear dynamic survival past extropy function.

In the following, by using the Equation (17), some ageing properties of random variable $X$ have been obtained. By differentiating Equation (17), we obtain

$$
\lambda_{Q}^{\prime}(u)=\frac{6 D S P J_{Q}^{\prime}(u)+2 u D S P J_{Q}^{\prime \prime}(u)}{\left(2 u D S P J_{Q}^{\prime}(u)+4 D S P J_{Q}(u)\right)^{2}} .
$$

Accordingly, the random variable $X$ is IFR if $D S P J_{Q}(u)$ is increasing and concave, while the random variable $X$ is DFR when $D S P J_{Q}(u)$ is decreasing and convex. Hereafter, some stochastic comparisons between two random variables $X$ and $Y$ with dynamic survival past extropy functions $D S P J_{Q_{X}}(u)$ 
and $D S P J_{Q_{Y}}(u)$, respectively have been assessed.

Corollary 3. If $X \leq_{R H Q} Y$ then $X \leq_{D S P Q J} Y$.

Proof. If $X \leq{ }_{R H Q} Y$, we conclude that $\frac{1}{p q_{X}(p)} \leq \frac{1}{p q_{Y}(p)}$ for all $0 \leq p \leq 1$. Therefore we have $\int_{0}^{u} p^{2} q_{X}(p) d p \leq \int_{0}^{u} p^{2} q_{Y}(p) d p$ and from Equation (15) the proof is completed.

This theorem concludes that whenever $X \leq_{R H Q} Y$, the system with lifetime $X$ is less reliable than that with lifetime $Y$ which also indicates that the information contained in the cumulative past lifetime distribution of $X$ is smaller than $Y$. The inverse of the Result 3 might not be true. Therefore, the circumstances that the inverse of the aforementioned theorem is valid, has been presented in the next theorem.

Corollary 4. Let $\frac{D S P J_{Q_{X}}(u)}{D S P J_{Q_{Y}}(u)}$ be the strictly increasing function in $u$. If $X \leq_{D S P Q J} Y$ then $X \leq_{R H Q} Y$.

Proof. With respect to $\frac{D S P J_{Q_{X}}(u)}{D S P J_{Q_{Y}}(u)}$ is the increasing function in $u$, we have

$$
\frac{u^{2} q_{X}(u) \int_{0}^{u} p^{2} q_{X}(p) d p-u^{2} q_{Y}(u) \int_{0}^{u} p^{2} q_{Y}(p) d p}{\left(\int_{0}^{u} p^{2} q_{Y}(p) d p\right)^{2}} \geq 0 .
$$

From the previous inequality, we conclude that $\frac{q_{X}(u)}{q_{Y}(p)} \geq \frac{\int_{0}^{u} p^{2} q_{X}(p) d p}{\int_{0}^{u} p^{2} q_{Y}(p) d p} \geq 1$ and the proof is completed.

Example 9. Assume that $Q_{X}(u)=u^{2}$ and $Q_{Y}(u)=u$. From Equation (15), we have $D S P J_{Q_{X}}(u)=-\frac{u}{3}$ and $D S P J_{Q_{Y}}(u)=-\frac{u}{6}$. It is obvious that $X \leq_{C P Q J} Y$. From Figure 2, it is clear that $X$ and $Y$ are not in reversed hazard quantile order. Moreover, $\frac{\operatorname{DSP}_{Q_{X}}(u)}{\operatorname{DSPJ}_{Q_{Y}}(u)}=2$ is constant function and so the condition given in Corollary 4 cannot be satisfied.

In the following, the relation between two orderings $\leq_{D S P J}$ and $\leq_{D S P Q J}$ is investigated and in the next examples, we show that the stochastic order $\leq_{D S P J}\left(\leq_{D S P Q J}\right)$ does not imply $\leq_{D S P Q J}\left(\leq_{D S P J}\right)$.

Example 10. Assume that $F(x)=x, 0 \leq x \leq 1$ and $G(y)=y^{2}, 0 \leq y \leq$ 1. From Equation (6), we have $D S P J_{F}(t)=-\frac{t}{6}$ and $D S P J_{G}(t)=-\frac{t}{10}$ therefore $X \leq_{D S P J} Y$. Moreover, let $Q_{X}(u)=u$ and $Q_{Y}(u)=u^{\frac{1}{2}}$ then from 


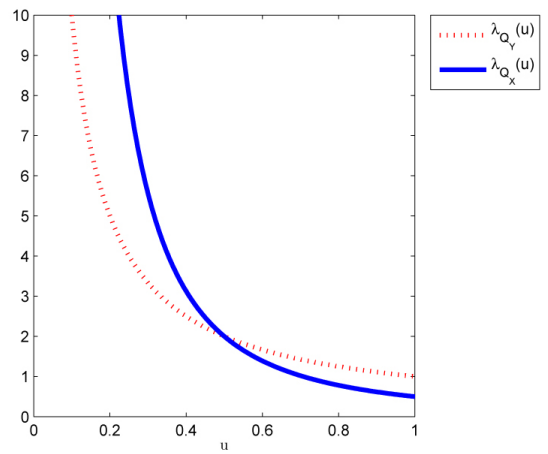

Figure 2. Plot of the reversed hazard quantile function.

Equation (15), we obtain $D S P J_{Q_{X}}(u)=-\frac{u}{6}$ and $D S P J_{Q_{Y}}(u)=-\frac{u^{\frac{1}{2}}}{10}$. Here, $D S P J_{Q_{X}}(u)$ and $D S P J_{Q_{Y}}(u)$ cross each other at $u=0.36$. Accordingly, ordering $\leq_{D S P Q J}$ does not exist between random variables $X$ and $Y$.

Example 11. Let $F(x ; c, R)=1-\left(1-\frac{x}{R}\right)^{c}, 0 \leq x \leq R$. From Table 1, we have

$$
\operatorname{DSPJ}_{F}(t ; c, R)=-\frac{t+\frac{2 c}{R}\left(1-\frac{t}{R}\right)^{c-1}\left(1-\left(1-\frac{t}{R}\right)^{c}\right)}{2\left(1-\left(1-\frac{t}{R}\right)^{c}\right)^{2}} .
$$

Left panel of Figure 3 shows that $D S P J_{F}(t ; 1,2)$ and $D S P J_{F}(t ; 2,2)$ are not ordered by $\leq_{D S P J}$. Moreover, $Q_{X}(u)=R\left(1-(1-u)^{\frac{1}{c}}\right)$ gives

$$
D S P J_{Q_{X}}(u ; c, R)=-\frac{R}{2 c u^{2}}\left[c-1-c(1-u)^{\frac{1}{c}}+\frac{c}{c+1}(1-u)^{\frac{1}{c}+1}\right] .
$$

From the right panel of Figure 3 , we observe that $D S P J_{Q_{X}}(u ; 1,2) \geq D S P J_{Q_{X}}(u ; 2,2)$.

Whereas $\leq_{D S P J}$ and $\leq_{D S P Q J}$ are not jointly inferred but they can make so under specific circumstances.

Corollary 5. Let DSPJ $J_{G}(t)$ is an increasing function in $t$ and $X \leq_{h r} Y$ or $X \leq_{r h r} Y$. If $X \leq_{D S P J} Y$ then $X \leq_{D S P Q J} Y$.

Proof. Since $X \leq_{D S P J} Y$ we conclude that $D S P J_{F}\left(Q_{X}(u)\right) \leq D S P J_{G}\left(Q_{X}(u)\right)$. From $X \leq_{h r} Y$ we have $Q_{X}(u) \leq Q_{Y}(u)$. As regards $D S P J_{G}(t)$ is an increasing function, we obtain $\operatorname{DSPJ}_{G}\left(Q_{X}(u)\right) \leq D S P J_{G}\left(Q_{Y}(u)\right)$ and the proof is complete. 

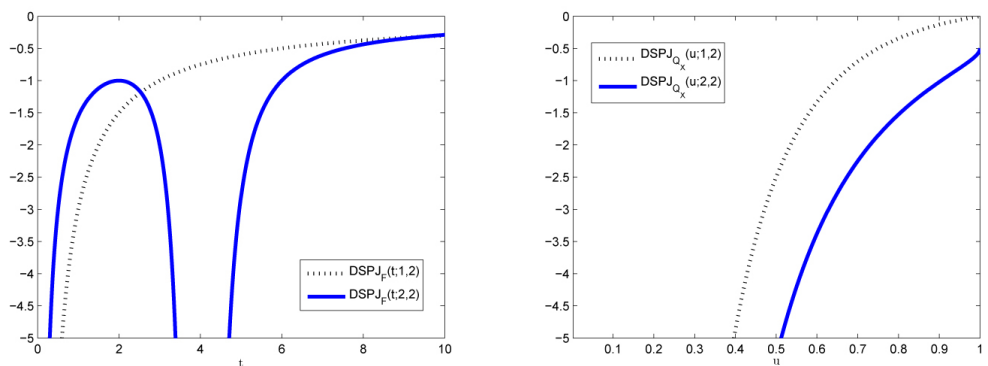

Figure 3. Plot of $D S P J_{F}(t ; 1,2), D S P J_{F}(t ; 2,2), D S P J_{Q_{X}}(u ; 1,2)$ and $D S P J_{Q_{X}}(u ; 2,2)$ in Example 11.

\section{Dynamic Survival Past Extropy of Parallel and Series Systems}

Consider a parallel (series) system with independent and identical components with lifetimes $X_{1}, \cdots, X_{n}$ with common cdf $F$. Let $X_{n: n}$ and $X_{1: n}$ be the smallest and largest order statistics from the random variables $X_{i}$, $i=1, \ldots, n$. The lifetime of parallel (series) system is $X_{n: n}\left(X_{1: n}\right)$; See Barlow and Proschan (1981). The dynamic survival past extropy of parallel system denoted by $\operatorname{DSPJ}_{F_{n: n}}(t)$ and is given by

$$
\begin{aligned}
\operatorname{DSPJ}_{F_{n: n}}(t) & =-\frac{1}{2} \int_{0}^{t}\left(\frac{F(x)}{F(t)}\right)^{2 n} d x \\
& =-\frac{1}{2 F(t)^{2 n}} \int_{0}^{\infty}(F(x))^{2 n} d x+\frac{1}{2} \int_{t}^{\infty}\left(\frac{F(x)}{F(t)}\right)^{2 n} d x \\
& =\frac{1}{F(t)^{2 n}} \mathrm{CJ}_{F_{n: n}}(t)+\frac{1}{2} \int_{t}^{\infty}\left(\frac{F(x)}{F(t)}\right)^{2 n} d x
\end{aligned}
$$

where $\mathrm{CJ}_{F_{n: n}}(t)$ indicates the cumulative extropy of parallel system.

Remark 1. For $t=\infty, \operatorname{DSPJ}_{F_{n: n}}(t)=\mathrm{CJ}_{F_{n: n}}(t)$.

In sequel, we provide a lower bound for the $\operatorname{DSPJ}_{F_{n: n}}(t)$.

Corollary 6. For all $t, \frac{\mathrm{CJ}\left(F_{n: n}\right)}{F^{2 n}(t)} \leq \operatorname{DSPJ}_{F_{n: n}}(t)$.

Proof. In Equation (25), it is clear that $\int_{t}^{\infty}\left(\frac{F(x)}{F(t)}\right)^{2 n} d x \geq 0$ and the proof is complete. 
Remark 2. Let $\mathrm{CJ}_{F_{1: n}}(t)$ is the dynamic survival past extropy of series system, then we have

(i) $\mathrm{CJ}_{F_{n: n}}(t) \geqslant n^{2} C J_{F}(t)$.

(ii) $\mathrm{CJ}_{F_{1: n}}(t) \geqslant n^{2} C J_{F}(t)$.

(iii) $\mathrm{CJ}_{F_{1: n}}(t) \geqslant-\frac{c n^{2}}{2}$, where $c=\int_{0}^{\infty} F(x) d x$.

Proof. The inequalities in part (i) and (ii) come directly from $F^{n}(x) \leq$ $n F(x)$.

Corollary 7. $\mathrm{DSPJ}_{F_{n: n}}(t)\left(\mathrm{DSPJ}_{F_{1: n}}(t)\right)$ is increasing (decreasing) function in $n$.

Let the sf of the random variable $t-X_{n: n} \mid X_{n: n}<t$ denoted by $\bar{F}_{n: n, t}$. It is obvious that

$$
\frac{\bar{F}_{n+1: n+1, t}}{\bar{F}_{n: n, t}}=\frac{F(x)}{F(t)}
$$

is less than one for $x \in(0, t)$ and so we have

$$
\left(t-X_{n+1: n+1} \mid X_{n+1: n+1}<t\right) \leq_{s t}\left(t-X_{n: n} \mid X_{n: n}<t\right) .
$$

Moreover, we have $\operatorname{DSPJ}_{F_{n: n}}(t)=-\frac{1}{2} \int_{0}^{t}\left(\bar{F}_{n: n, t}(x)\right)^{2} d x$, therefore the proof is completed. For $\operatorname{DSPJ}_{F_{1: n}}(t)$, the proof is obtained in a similar way.

Example 12. Let the identical random variables $X_{1}, \cdots, X_{n}$ delineated via exponential distribution with mean $1 / \lambda$. In Figure 3 , for $t=2$ and $\lambda=0.2$ the dynamic survival past extropy of $X_{n: n}$ and $X_{1: n}$ is depicted for $n=1, \ldots, 10$ in left and right panel, respectively. Figure 3 shows that $\operatorname{DSPJ}_{F_{n: n}}(2)\left(\operatorname{DSPJ}_{F_{1: n}}(2)\right)$ is increasing (decreasing) in $n=1, \ldots, 10$ and confirms Result 3.

Proposition 1. Let $X$ and $Y$ be two positive random variables with pdfs $g(x)$ and $h(x)$ and absolutely continuous cdfs $G(x)$ and $H(x)$, respectively. Then $G$ and $H$ belong to the same family of distributions, but for a change in location and scale, if and only if for $t>0, \operatorname{DSPJ}_{F_{n: n}}=\operatorname{DSPJ}_{G_{n: n}}$, for $n=n_{j}, j \geq 1$ such that $\sum_{j=1}^{+\infty} \frac{1}{n_{j}}$ is infinite.

Proof. First assume that $\operatorname{DSPJ}_{F_{n: n}}=\operatorname{DSPJ}_{G_{n: n}}$. Then, Equation (25) implies that

$$
\int_{0}^{1} u^{2 n}\left(\frac{F(t)}{f\left(F^{-1}(u F(t))\right)}-\frac{G(t)}{g\left(G^{-1}(u G(t))\right)}\right) d u=0
$$



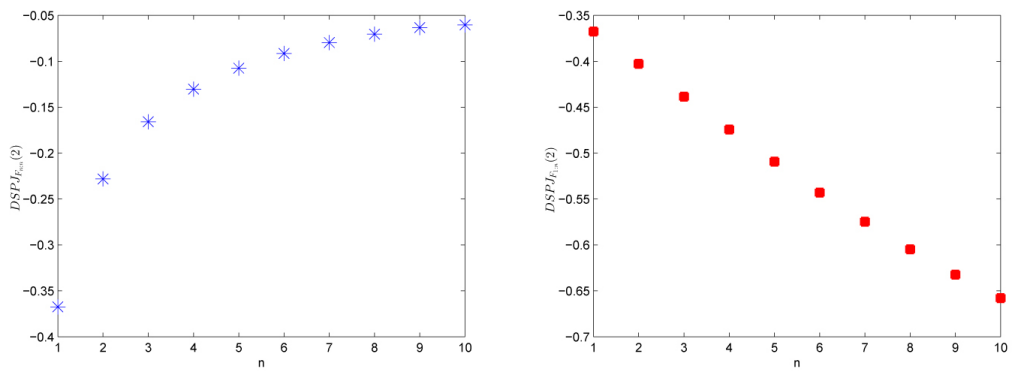

Figure 4. Plot of dynamic survival past extropy of $X_{n: n}$ and $X_{1: n}$ for $n=1, \ldots, 10$ in left and right panel, respectively.

holds for $j \geq 1, n=n_{j}$, such that $\sum_{j=1}^{+\infty} \frac{1}{n_{j}}=\infty$. By Muntz-Szasz Theorem, we conclude that $f\left(F^{-1}(u F(t))\right)=g\left(G^{-1}(u G(t))\right)$, for $u \in(0,1)$. On the other hand, since $d F^{-1}(u F(t)) / d v=\left(f\left(F^{-1}(t)\right)\right)^{-1}$, we have $d F^{-1}(u F(t)) / d v=$ $d G^{-1}(u G(t)) / d v, t \in(0,1)$. It then follows that $F^{-1}(u F(t))=G^{-1}(u G(t))+$ $k, u \in(0,1)$, i.e. $F^{-1}\left(u^{\prime}\right)=G^{-1}\left(u^{\prime}\right)+k, u^{\prime} \in(0,1)$. This means that $F$ and $G$ belong to the same family of distributions, but for a possible change in location. The necessity is trivial.

\section{Conclusion}

In this paper, we investigate the dynamic survival past extropy and the cumulative extropy as the measure of uncertainty. The information properties of these extropies have been studied in details. Some stochastic comparisons and bounds are obtained and for parallel and series systems the performance of the aforementioned informations are assessed. Also, the quantile-based dynamic survival past extropy approach is considered.

\section{References}

Alizadeh Noughabi, H. and Jarrahiferiz, J. (2019). On the Estimation of Extropy. Journal of Nonparametric Statistics, 31, 88-99.

Barlow, R. and Proschan, F. (1981). Statistical Theory of Reliability and Life Testing, Probability Model. New York, Holt, Rinehart and Winnston.

Cover, T.M. and Thomas, J.A. (2012). Elements of Information Theory. John Wiley and Sons. 
Jahanshahi, S.M.A., Zarei, H. and Khammar, A.H. (2019). On Cumulative Residual Extropy. Probability in the Engineering and Informational Sciences, 34, 1-21.

Jose, J. and Sathar, E.A. (2019). Residual Extropy of k-Record Values. Statistics and Probability Letters, 146, 1-6.

Krishnan, A.S., Sunoj, S.M. and Nair, N.U. (2020). Some Reliability Properties of Extropy for Residual and Past Lifetime Random Variables. Journal of the Korean Statistical Society, 49, 457-474.

Lad, F., Sanfilippo, G. and Agro, G. (2015). Extropy: Complementary Dual of Entropy. Statistical Science, 30, 40-58.

Midhu, N.N., Sankaran, P.G. and Nair, N.U. (2013). A Class of Distributions with the Linear Mean Residual Quantile Function and It's Generalizations. Statistical Methodology, 15, 1-24.

Nanda, A.K., Sankaran, P.G. and Sunoj, S.M. (2014). Renyi's Residual Entropy: A Quantile Approach. Statistics and Probability Letters, 85, 114-121.

Noughabi, H.A. and Jarrahiferiz, J. (2020). Extropy of Order Statistics Applied to Testing Symmetry. Communications in Statistics-Simulation and Computation, 1-11.

Qiu, G., Wang, L. and Wang, X. (2019). On Extropy Properties of Mixed Systems. Probability in the Engineering and Informational Sciences, 33, 471-486.

Qiu, G. (2019). Further Results on Quantile Entropy in the Past Lifetime. Probability in the Engineering and Informational Sciences, 33, 146-159.

Qiu, G. and Jia, K. (2018a). The Residual Extropy of Order Statistics. Statistics and Probability Letters, 133, 15-22.

Qiu, G. and Jia, K. (2018b). Extropy Estimators with Applications in Testing Uniformity. Journal of Non-parametric Statistics, 30, 182-196.

Qiu, G. (2017). The Extropy of Order Statistics and Record Values. Statistics and Probability Letters, 120, 52-60.

Shaked, M. and Shanthikumar, J. (2007). Stochastic Orders. Springer, New York.

Shannon, C.E. (1948). A Mathematical Theory of Communication. The Bell System Technical Journal, 27, 379-423.

Sunoj, S.M. and Sankaran, P.G. (2012). Quantile based Entropy Function. Statistics and Probability Letters, 82, 1049-053.

Sunoj, S.M., Krishnan, A.S. and Sankaran, P.G. (2017). Quantile-based Entropy of Order Statistics. Journal of the Indian Society for Probability and Statistics, 18, 1-17. 
Yang, J., Xia, W. and Hu, T. (2018). Bounds on Extropy with Variational Distance Constraint. Probability in the Engineering and Informational Sciences, 33, 1-19.

Yu, H. L. and Wang, C.H. (2013). Quantile-based Bayesian Maximum Entropy Approach for Spatiotemporal Modeling of Ambient Air Quality Levels. Environmental Science and Technology, 47, 1416-1424.

\section{Zohreh Pakdaman}

Department of Statistics, University of Hormozgan,

Bandarabbas, Iran.

email: zpakdaman@hormozgan.ac.ir

\section{Majid Hashempour}

Department of Statistics, University of Hormozgan,

Bandarabbas, Iran.

email: ma.hashempour@hormozgan.ac.ir 